\title{
Development and Manufacturing of a controlled 3D printed bionic hand
}

\author{
H. Smajic 1[https://orcid.org/0000-0002-0669-1979], T. Duspara 1[https://orcid.org/0000-0002-6985-5950] \\ ${ }^{1}$ Technische Hochschule Köln
}

\begin{abstract}
During a current project, a fully functioning prototype of a 3D printed bionic hand was developed. This paper explains principles such as: bionic hand movement, working rules of sensors and actuators etc. Design of all parts are performed, including the wiring of control system. The project includes two types of sensor control systems for bionic hand. One is with stretch sensors that replicates movement of human hand onto the bionic model. Other type is using machine learning $(\mathrm{Al})$ and a camera. The average amputee cost is $\$ 30.000,00$ for a new custom-built arm/hand. With the advancement of technology through time, manufacturing processes became cheaper and more accessible. Technical innovation of this project was the fact, that a functional prosthetic hand prototype was built for price lower than $\$ 50,00$. The prototype does not have all the functions and capabilities as the full priced custom prosthetic hand, but it can replicate altogether the movements as the real device. All the fingers are capable of moving individually, sideways and with the work on the new version, gripping function could be perfected. Further work on materials, could help find the adequate material to increase friction and thusly enhance the grasp strength. The new challenge would involve testing with different kinds of materials to improve the working stability. As it was already unfavorable, this project was mostly based onto the actuation part, or rather the hand itself. Second part of research would involve exploring of different sensor systems. Two control solutions were designed and tested. Next steps would involve neurotransmission sensors, where arm would be controlled using brainwaves as signals that are transformed in movement.
\end{abstract}

Keywords: bionic hand, Al, 3D printing, low-budget

\section{Introduction}

Main goal while building a prosthetic hand is to replicate all the movements as a human hand has. To achieve desired movement, all bones of a human hand must be replicated and designed. 3D printed prosthetic hands are well known in $21^{\text {st }}$ century but unfortunately, they are not widely used because of durability of the $3 \mathrm{D}$ printed material. With further advancement of technology, improvements of composite materials are inevitable.

\section{Mechanical parts}

A human hand consists of three basic bone groups. Those are phalanges, metacarpals and carpals. To design a 3D model of a hand, same bone parts should be engineered. Those are distal phalanx, middle phalanx and proximal phalanx. The main function of hand can be recreated and engineered using only phalanges, so metacarpal and carpal bones will be merged into a one piece. 
Fingers (Phalanges) - Distal phalanx is the tip of the finger. On a 3D model of this part, a pre designed holes can be found. These include holes for the shaft, and grooves for the strings. Going from up to down, next part is the middle phalanx which is placed into the distal phalanx and secured with said shaft. On the top and the bottom of middle phalanx there are enter and exit holes for the string. Proximal phalanx represents the lowest phalanx bone. This is the last moving part of the bionic hand. It is the largest phalanx bone with the maximum length of approximately $60 \mathrm{~mm}$. It is connected to the rest of the hand using a connector and two shafts. The upper shaft is the same as with the two earlier mentioned bones. Lower shaft connects it to the central part of the hand which represents the merged metacarpal and carpal bones. The difference between two shafts is the freedom of movement. Upper shaft allows the piece to move towards and away from the palm. Lower shaft allows the movement of whole finger in a sideways direction. Because phalanges are connected to each other, movement of proximal phalanx sideways results in the movement of whole finger. Unlike the other fingers thumb consists of only two parts but it has the ability to move into two different directions. Bottom part of the thumb is actually a holder for a servo motor that rotates the thumb around its nominal axis. As well as with other fingers, string is placed through the thumb thus achieving the regular movement towards the palm, or in other

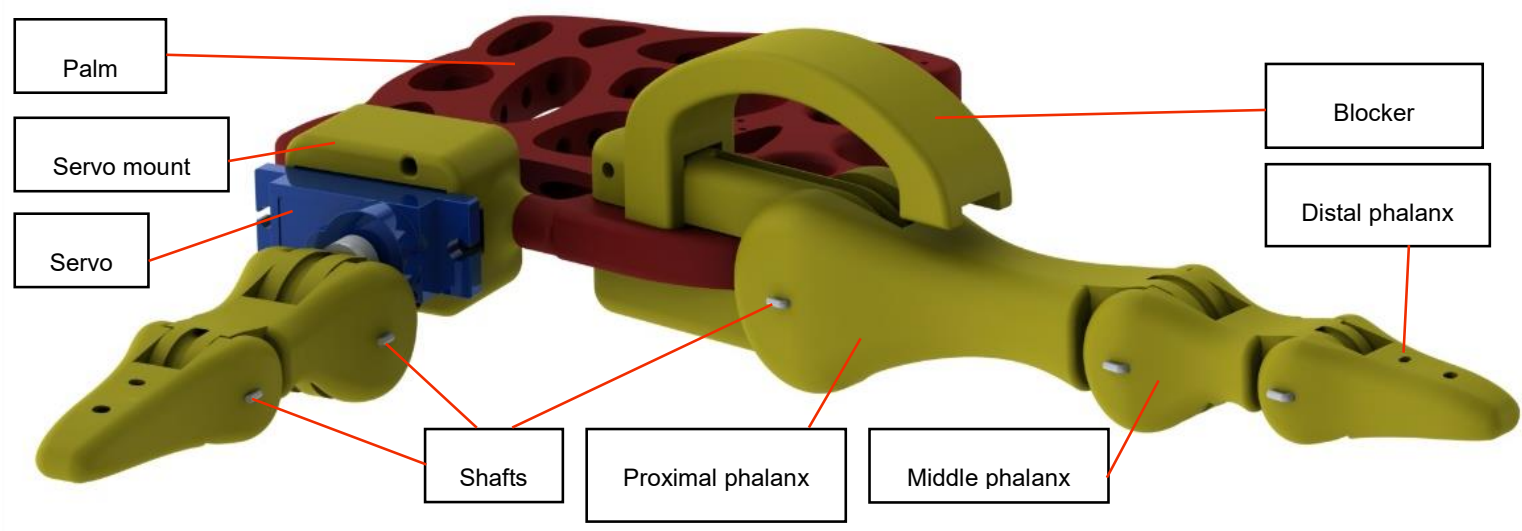

Figure 1. Phalanges Assembly.

words, the "grip“ itself.

Palm - As the movement of metacarpal and carpal bones is minimal, they will be replaced with a single piece that represents the palm of a bionic hand. Each finger has one string with two endpoints. Pulling one endpoint moves the finger towards the palmand other endpoint moves that guide the strings. It is crucial to design the blockers for each individual finger. These blockers are placed on the back side of the finger-palm connectors. When the finger goes from fully the finger back into the first position. To avoid confusion and mess, the palm has holes closed to fully open position, the proximal phalanx hits the blocker and stays in place.

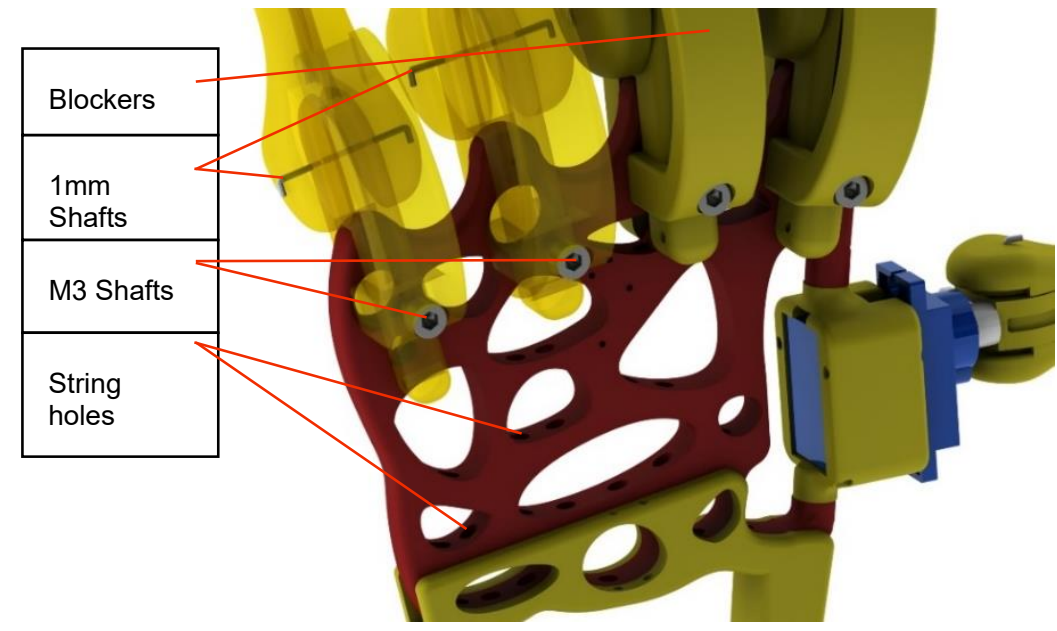

Figure 2. Palm Assembly. 
Arm and stand design - While designing the arm the crucial thing to do is to provide slots for the servo motors. Arm model is compact and has slots for six servo motors. To avoid the collision between levers of servo motors, half of the servos are facing the front side and the other half are facing the back side. The stand is placed at the bottom of the hand. To lower the center of mass, and prevent falling of the hand due to movement inertia, a $0,5 \mathrm{~kg}$ weight is added to the stand. The lower part of the arm fits snuggly into the stand. Stand is the last part of the mechanical assembly. In order to test the prototype, a housing was designed, which fits all the electronical components.

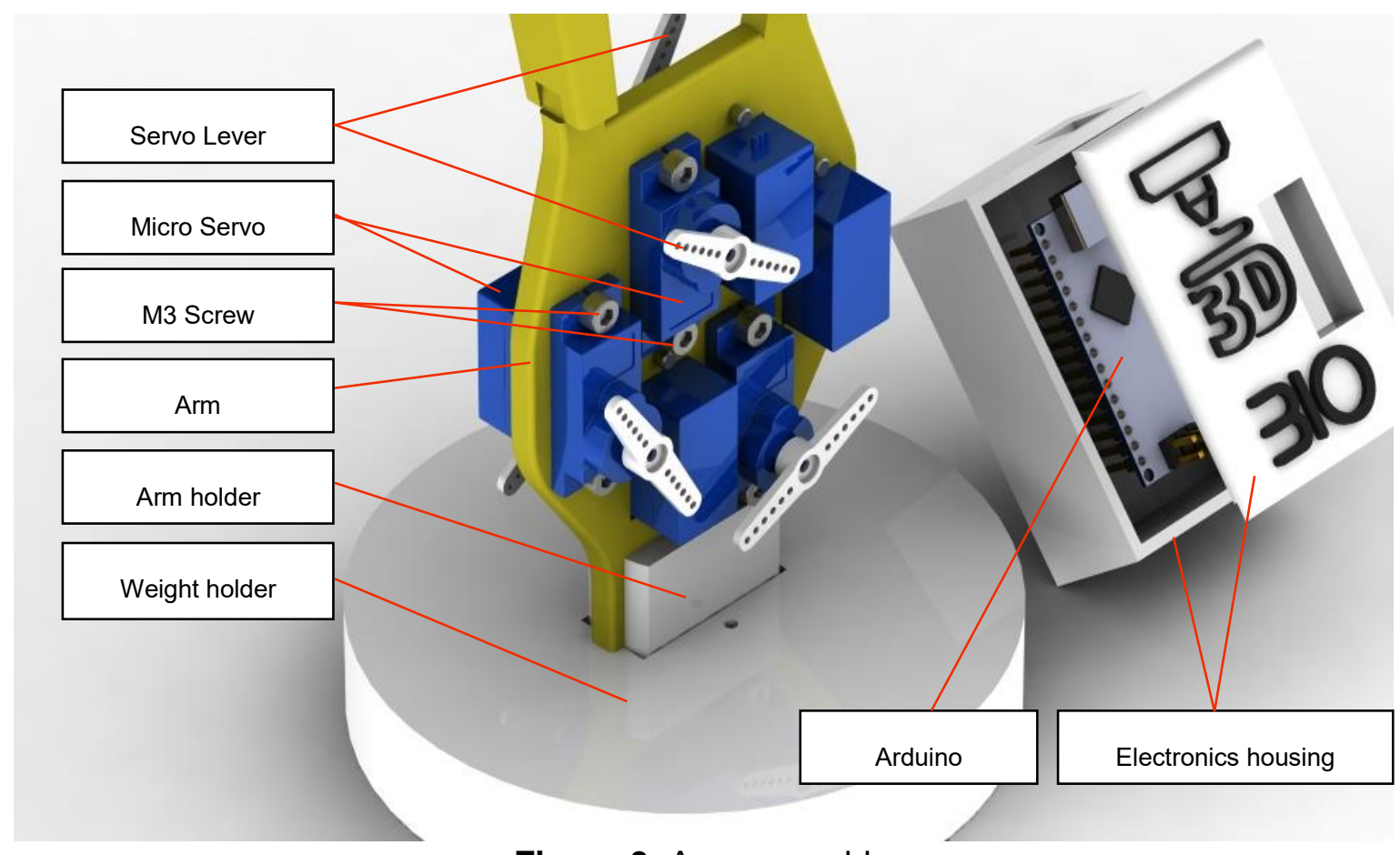

Figure 3. Arm assembly.

Strings - For the movement of fingers or in other word the connection between phalanges and servo motor, a fishing nylon is used. The one used for prototype is $0.2 \mathrm{~mm}$ thick and it has the load strength of $20[\mathrm{~N}]$ which is more than enough to support the force required to achieve movement of the hand. Each piece of nylon string is cut to the size of approximately $600 \mathrm{~mm}$. This string is pulled through all of the phalanges in a way that the two endpoints beneath the arm are equally long. The center of string is glued on top of the distal phalanx of each finger. Later on the string goes through the holes inside the palm and finally through the arm-palm connector and to the servo motors placed on the arm. The string is pulled through holes on servo levers and secured with adhesive. Before securing the string itself, a proper movement should be checked. If everything is moving according to the plan, the strings can be attached onto the lever. For entire assembly, less than $3 \mathrm{~m}$ of nylon are needed. Nylon is very cheap so material cost does not affect overall price.

\section{Actuators}

Actuators are devices that provide movement for the assembly. Micro servomotors are chosen because of their compact design and easy control. A servomotor is a rotary or linear actuator that allows for precise control of angular or linear position, velocity and acceleration. They can easily be mounted onto the arm part. For the full movement of the hand, seven servomotors are required. Five motors enable the movement of five fingers where the sixth motor enables the rotational movement of the thumb. The seventh motor is used for sideways movement of fingers. Servomotors can rotate their shaft for 180 degrees. The servo lever should be around $35 \mathrm{~mm}$ long. If the lever is shorter, the finger doesn't reach the end positions. There are two positions of the motors that are used for the movement. The 180-degree position places the finger fully open and 0 degree moves the finger back into its 
first point. Servomotors used for prototype have metal gears inside them which makes them more durable. Another benefit from these motors is their price. For achieving the basic movements, seven servo motors are needed which in the end, adds up to the price of $19,99 €$.

\section{Production process}

Process of building a hand can be classified in five phases:

1. CAD/CAM Design

2. Manufacturing

3. Quality control

4. Assembly

5. Automation

\section{CAD/CAM Design}

Phase one includes scaling of the parts, so they can perfectly fit individual person. Each part needs to be scaled by certain percentage depending on the size of the hand. Scaling is done using a CAD software (SolidWorks, NX, etc.). After the scaling is done, an animation must be ran. Animation shows potential problems and irregularities without the necessity for a prototype. When animation shows solid work and movement of all the parts, each part needs

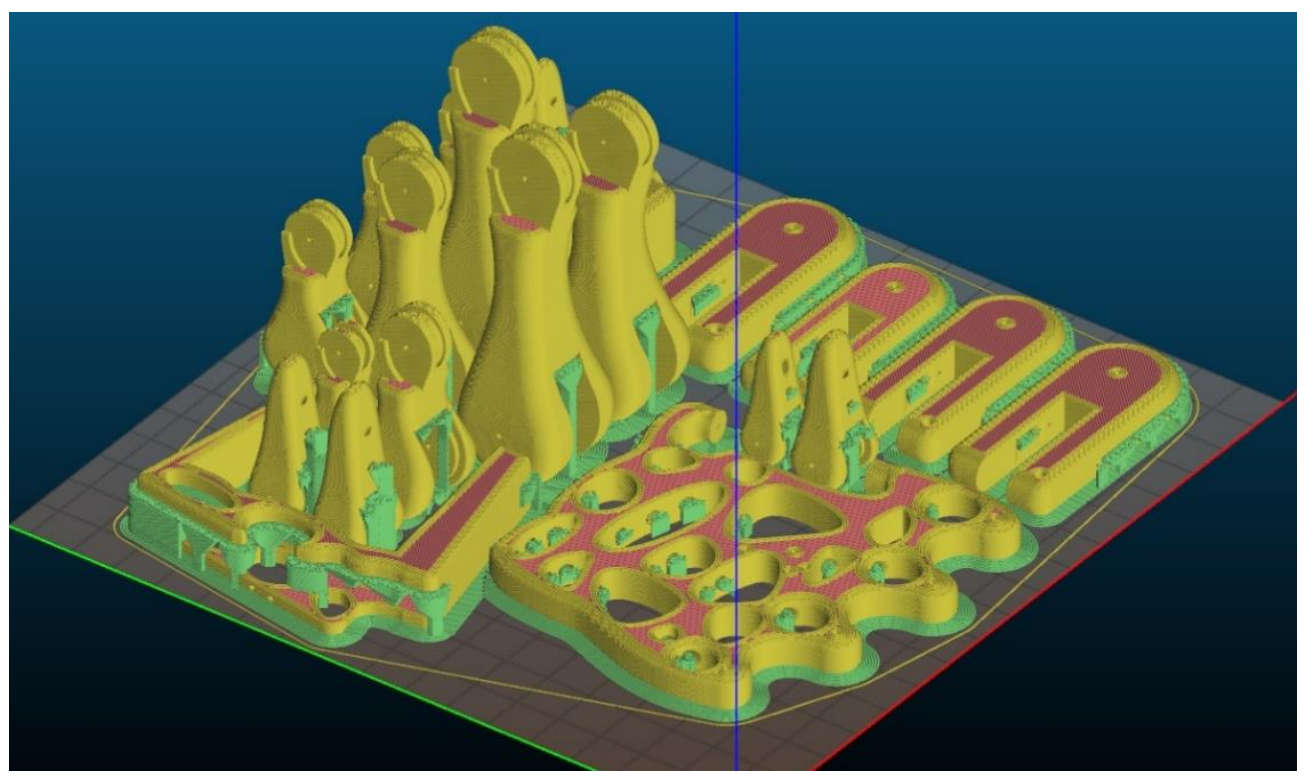

Figure 4. CAM Preparation.

to be converted into triangulated surface-s commonly known as STL files. Converted files are then imported into a CAM software. Software used is called "Slic3r" and it is utilized for 3D printer CAM preparation. CAM software takes the STL files, and later on computes the coordinates used for the printer movement, based on selected parameters. Parameters include: print, printer and material specifications. Most important are the print specifications, whereas other two depend on the specific machines that are used. Parts need to be manipulated and positioned on the print bed in a way that conserves the space on the printing surface. After thorough calibration and testing, the ideal parameters can be chosen. The ones chosen for this particular print and printer are shown in table below. 
Table 1. Printing parameters.

\begin{tabular}{|c|c|c|c|c|}
\hline & \multicolumn{2}{|l|}{ Parameter } & Value & Unit \\
\hline \multirow{10}{*}{ 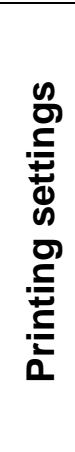 } & \multicolumn{2}{|c|}{ Layer height } & 0.16 & $\mathrm{~mm}$ \\
\hline & \multicolumn{2}{|l|}{ Fill density } & $50 \%$ & \\
\hline & \multicolumn{2}{|l|}{ Fill pattern } & Triangle & \\
\hline & \multirow{3}{*}{ Printing speed } & Outer perimeters & 20 & $\mathrm{~mm} / \mathrm{s}$ \\
\hline & & Other perimeters & 25 & $\mathrm{~mm} / \mathrm{s}$ \\
\hline & & & 30 & $\mathrm{~mm} / \mathrm{s}$ \\
\hline & \multicolumn{2}{|l|}{ Skirt } & 1 & loops \\
\hline & \multicolumn{2}{|l|}{ Brim } & 5 & $\mathrm{~mm}$ \\
\hline & \multicolumn{2}{|c|}{ Generate support material: } & YES & \\
\hline & \multicolumn{2}{|c|}{ Overhang threshold: } & $65 \%$ & \\
\hline
\end{tabular}

\section{Manufacturing}

When the parameters are all set, CAM software generates G-code used for the print. Parts are made out of $A B S$ filament with $50 \%$ infill. Filament has $1.75 \mathrm{~mm}$ diameter, so it is easy to calculate its mass. One spool of ABS material contains 290,7 meters of filament (1 $\mathrm{kg} \mathrm{spool}$ ). Printers surface has printable area of $20 \times 20 \mathrm{~cm}$. In regard to that, two separate G-Code files must be created to fit all the parts of a bionic hand. Printing process for entire hand uses $89,5 \mathrm{~m}$ of ABS filament which weighs $308 \mathrm{~g}$. The main benefit behind this project is its "Lowcost factor". ABS filament spool costs around 20,00€, so mechanical parts can be manufactured with this technology for only $4,52 €$. This means, that you can create all the mechanical moving parts of a bionic hand for under $5,00 €$ thanks to the advancement of manufacturing processes and technologies.

\begin{tabular}{|l|l|l|l|l|l|l|}
\hline Print No. & Pieces & $\begin{array}{l}\text { Filament } \\
\text { length }[\mathbf{m}]\end{array}$ & $\begin{array}{l}\text { Fillament } \\
\text { mass [g] }\end{array}$ & $\begin{array}{l}\text { Layer } \\
\text { count }\end{array}$ & $\begin{array}{l}\text { Print } \\
\text { time }\end{array}$ & $\begin{array}{l}\text { Cost } \\
{[\boldsymbol{\epsilon}]}\end{array}$ \\
\hline Print 1 & 22 & 59,242 & 203,78 & 1107 & $22: 24: 33$ & 2,99 \\
\hline Print 2 & 13 & 30,271 & 104,12 & 312 & $07: 59: 01$ & 1,53 \\
\hline Altogether & $\mathbf{3 5}$ & $\mathbf{8 9 , 5 1 3}$ & $\mathbf{3 0 7 , 9 1}$ & $\mathbf{1 4 1 9}$ & $\mathbf{3 0 : 2 3 : 3 4}$ & $\mathbf{4 , 5 2}$ \\
\hline
\end{tabular}

Table 2. Material expenses.

According to the table above and earlier mentioned price of servomotors, overall assembly worth does not exceed $25,00 €$. In abstract there was a 50,00€ price mentioned for the assembly. This price consists of labor (staff), disposable material, screws etc.

\section{Quality control}

After the manufacturing process, which takes a little more than 30 hours, it is time to clean the parts and check dimensions. Support material must be removed from surfaces, and surfaces should be grinded. In order to achieve perfect clearance in between parts, all the dimensions must be checked and compared to the dimensions in CAD drawing. If some of the dimensions are unequal to the ones in the drawing, they must be corrected. Perfect gap between two parts can be tested after adding a shaft between them. If the parts move one around another smoothly and without any problems collide with their limit stops (blockers), then the clearance is fine and shaft can be fixed in place. If the movement seems too rough, then contact surfaces need to be grinded $0,2 \sim 0,4 \mathrm{~mm}$. This process is repeated for each connecting part until the clearance is satisfactory. 


\section{Assembly}

Assembly phase starts after acquisition of all parts and components. Micro servomotors are placed into slots on the arm and secured in place with M3 screws. To extend servo levers, new longer levers were made and placed onto the existing ones. When all the mechanical parts are printed and dimensions are checked, all the parts should be lined up one next to another. Next phase is pushing the string through predesigned grooves on the phalanx's parts. This process requires a lot of patience.

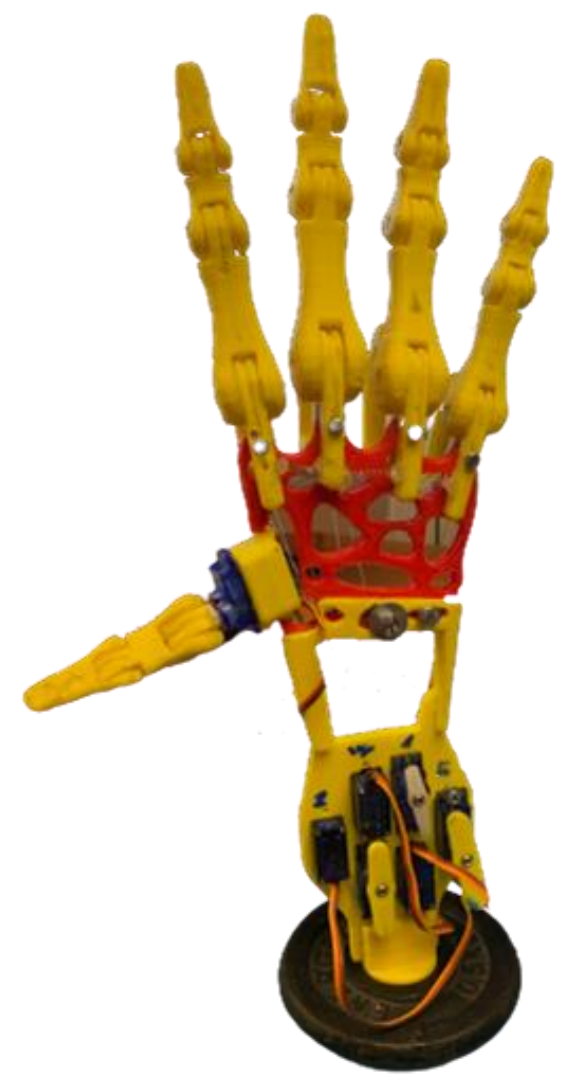

Figure 5. Full assembly of a bionic hand.

The grooves are very narrow and due to the imperfections of printing material, it is common for the strings to stick. After all the strings have been pulled through phalanges and palm, the shafts are mounted. At this point, string ends are equal length and they are secured at the top of distal phalanx with glue. Pulling the two endpoints will result in opening or closing fingers. If the movement is as desired, endpoints must be linked with servo levers. Before firmly securing string to servo levers, movement is checked by carefully rotating the axis of servomotor. Zero-degree position results in fully open finger whereas 180-degree position results in fully closed finger. When all the movements are examined and approved, strings are secured to servo levers with adhesive and leftover can be cut off.

\section{Automation}

With fully assembled hand, the means of control must be constructed. Control system requires a microcontroller that moves servomotors. Microcontroller used in this project is an Arduino Nano microcontroller. Arduino nano has 13 digital I/O pins. For control of the actuators, each servo requires one PWM output pin. That means that seven output pins are used for the servo movement. Generally Arduino can supply voltage to actuate these servos, but because there are seven servos attached to this assembly, Arduino voltage output cannot provide enough current to move all of them simultaneously. Additional power source is added to raise the current in servos. For this project a $20 \mathrm{~W}$ power source is used. This provides servos with $5 \mathrm{~V}$ of voltage and 4 Ampers of current. To connect all the servos to Arduino, a custom PCB bord was designed. On one end of the board there is a standard 
voltage connector. The plus pin of voltage connector is soldered to the VIN pin of Arduino. This provides the power that Arduino needs to operate without additional power source (USB). The same pin is connected to all of the middle pins of servo connector. Other pin is soldered to the Arduino GND pin. With this, all the devices including servos and Arduino are powered with the same power source. The USB connector on the Arduino is only used to transfer the code from the computer to the microcontroller.

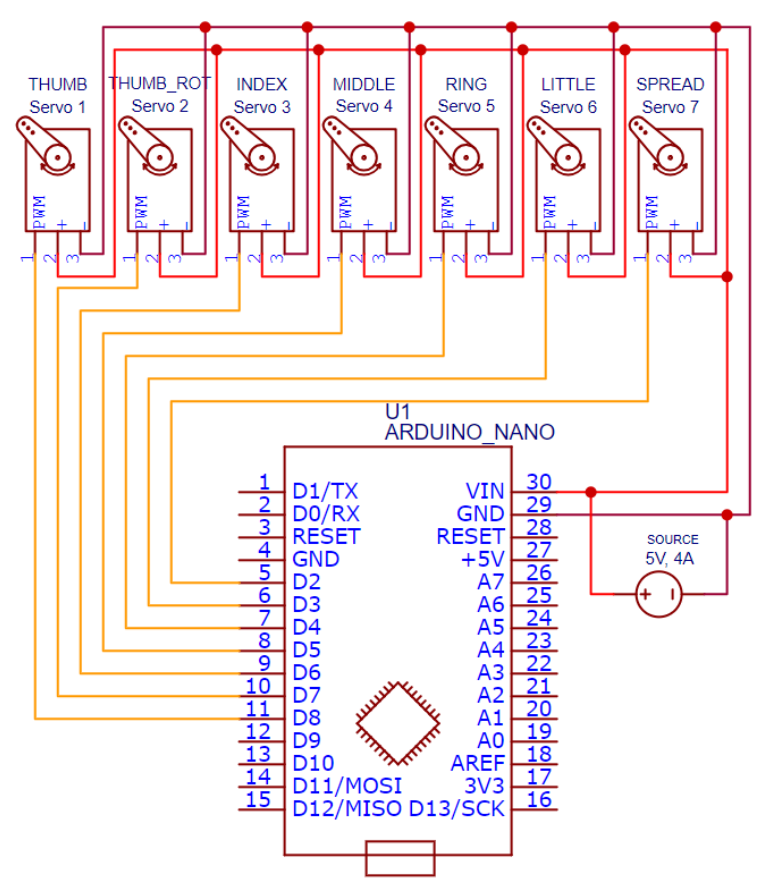

Figure 7. Wiring schematic.

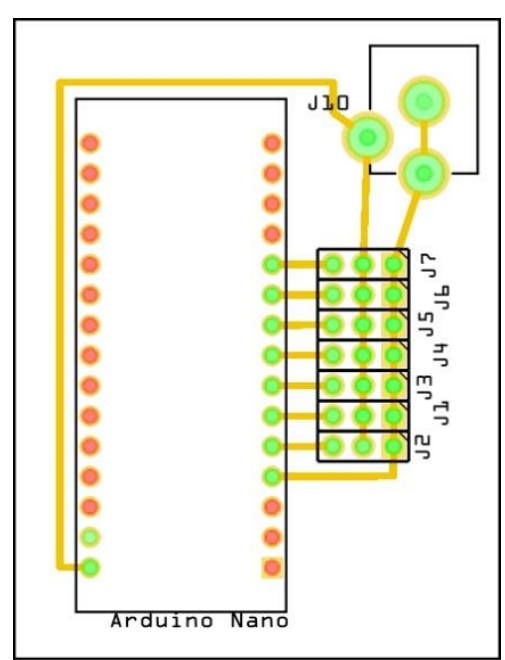

Figure 6. PCB Design.

\section{Conclusion}

During the work on this project, a final prototype was built. The average amputee pays tenths of thousands of Euros for a new custom-built arm/hand. With the advancement of technology through time, manufacturing processes became cheaper and more reachable. Technical innovation of this project was the fact, that a functional prosthetic hand prototype was built for under $30,00 €$. This prototype does not have all the functions and proficiency as the full priced prosthetic hand, but it can replicate all the movements as the real device. All the fingers are capable of moving individually, sideways and with the work on the new version, gripping function could be perfected. Further work on materials, could help find the adequate material to increase friction and thusly enhance the grasp strength. Further work on this project would involve experimentation with different kinds of materials to improve the operational stability. As it was already said, this project was mostly based onto the actuation part, or rather the hand itself. Second part of research would involve exploring of different sensor systems. Interesting potential was noticed in works with neurotransmission sensors. Next phase could involve synthesis of a low budget 3D printed bionic hand with said sensors.

\section{References}

1 Taylor C, Scwarz R. The Anatomy and Mechanics of the Human Hand. Artificial Limbs . 1955;2(2):22-35.

2 Shi W, Lyu Z, Tang S, Chia T, Yang C. A bionic hand controlled by hand gesture recognition based on surface EMG signals: A preliminary study. Biocybernetics and Biomedical Engineering. 2018;38(1):126-135.

https://doi.org/10.1016/j.bbe.2017.11.001 
3 Bronzino J, Peterson D. Biomedical engineering fundamentals. 2nd Edition. CRC Press; 2014. https://doi.org/https://doi.org/10.1201/b15482

4 Clement R, Bugler K, Oliver C. Bionic prosthetic hands: A review of present technology and future aspirations. The Surgeon. 2011 Dec;9(6):336-340. https://doi.org/10.1016/j.surge.2011.06.001

5 Aman M, Sporer ME, Gstoettner C, Prahm C, Hofer C, Mayr W, Farina D, Aszmann OC. Bionic hand as artificial organ: Current status and future perspectives. Artificial Organs. 2019 02;43(2):109-118. https://doi.org/10.1111/aor.13422

6 Syed A, Agasbal ZTH, Melligeri T, Gudur B. Flex Sensor Based Robotic Arm Controller Using Micro Controller. Journal of Software Engineering and Applications. 2012;05(05):364-366. https://doi.org/10.4236/jsea.2012.55042 